\title{
Psychophysiologic Correlates of Unconsciousness and Near-Death Experiences
}

\author{
James E. Whinnery, Ph.D., M.D. \\ West Texas Agricultural and Mechanical University, \\ Canyon, TX
}

\begin{abstract}
Although loss and recovery of consciousness are integral parts of the near-death experience (NDE), the role of altered states of consciousness in the NDE has not been thoroughly investigated. Sixteen years of observations, including nearly 1000 acceleration $(+\mathrm{Gz})$-induced loss of consciousness (G-LOC) episodes, have revealed a pattern of psychophysiologic symptoms that collectively constitute the G-LOC syndrome and relate to the kinetics of $+\mathrm{Gz}$-induced ischemia. Comparison of the psychophysiologic symptoms of G-LOC episodes and NDEs may help identify those symptoms unique to near-death and the process of dying. I propose a classification scheme for the study of NDEs and for relating near-death research to other psychophysiologic research. This scheme is based on the neurologic states of the nervous system and the transitions between them that result from alteration of blood flow to the nervous system. Consciousness and unconsciousness are considered as the neurobiologic bases of a balance between survival in the external environment and protection from ischemic threat within the internal environment. The study of G-LOC episodes in healthy individuals is therefore of value for understanding the mechanisms of both loss of consciousness and the NDE.
\end{abstract}

You cannot die without losing consciousness. Everyone should, therefore, have some interest in loss of consciousness, since he or she will experience it at least once. As such, unconsciousness is a

James E. Whinnery, Ph.D., M.D., is on the Science Advisory Board of the National Institute for Discovery Science, and is Professor of Chemistry at West Texas Agricultural and Mechanical University. Reprint requests should be addressed to Dr. Whinnery at the Department of Chemistry, West Texas A \& M University, Canyon, Texas 79019-0001. 
universal human event, albeit possibly only a terminal one for some. Death is covered by the physiologic veneer of unconsciousness. The common pathophysiologic pathway leading to death of the nervous system and therefore the human body involves loss of consciousness. In support of operational requirements, fighter aviation medical research has the opportunity to investigate experimentally loss of consciousness in healthy humans and, therefore, provide information on this universal experience. I share these loss of consciousness results so that those interested in understanding the process of dying and near-death experiences (NDEs) may utilize them to isolate carefully the components of those phenomena that occur only as a result of losing and regaining consciousness.

Although history and scientific research efforts are replete with inquiry into the secrets of death, our understanding of the psychophysiologic aspects of the dying process remains relatively limited. Of interest have been the questions relating to close encounters with death and an existence after death. These have been topics for philosophers, theologians, parapsychologists, and clinicians. Recent studies have focused on evaluating individuals who have experienced NDEs, survived "sudden death" episodes, and had other types of lifethreatening experiences. In spite of the recurrent psychophysiologic findings from these studies, little emphasis has been placed on the common physiologic event that occurs: loss of consciousness. The NDEs and "sudden death" episodes require that loss and recovery of consciousness be at least a portion of the event. It is therefore evident that a detailed understanding of unconsciousness should be considered an integral part of the scientific investigation of such events. The results of loss of consciousness experiments in completely healthy humans may provide insight into the normal neurologic processes that occur in association with NDEs and "sudden death" episodes.

This report focuses on the psychophysiologic events associated with acceleration $(+\mathrm{Gz})$-induced loss of consciousness (G-LOC) in completely healthy individuals. Head-to-foot acceleration $(+\mathrm{Gz})$ stress is a unique aspect of flying fighter aircraft engaged in aerial combat maneuvering. $+\mathrm{Gz}$ stress reduces blood flow to the head and causes pooling of blood in the abdomen and extremities. Cephalic nervous system ischemia results in G-LOC. Since 1919, G-LOC has remained a perplexing operational problem in fighter aircraft aviation that is challenging fighter aviation medical specialists to attain a solution, in order to reduce the loss of highly trained aircrew and multimil- 
lion-dollar aircraft that continues to occur (Burton, 1988). A solution for the G-LOC problem requires a thorough understanding of the alterations of consciousness. Although preventing further losses of aircrew and aircraft is the goal of fighter aviation medicine, the results from experiments involving loss of consciousness in completely healthy humans should be of interest to a broad range of scientific disciplines. Our research does not seek to explain, nor is this report presented to explain, the totality of NDEs or "sudden death" episodes. Since we do have the unique opportunity routinely to collect data involving loss of consciousness in healthy humans, we present it to illustrate at least some of the phenomena that would be expected in association with NDEs and "sudden death" episodes that also involve loss of consciousness.

\section{Methods}

For those not familiar with aerial combat maneuvering in fighter aircraft and ground-based human centrifuge research, the stresses on the human body that result from $+\mathrm{Gz}$ stress can be understood as analogous to what happens in a test tube of blood when it is placed in a small clinical laboratory centrifuge to separate the more dense red blood cells from the plasma. The red blood cells are displaced to the bottom (foot) of the test tube (body) away from the top (head) of the test tube. In fighter aircraft or in a human centrifuge it is not just the red blood cells that are displaced; it is the blood in general that is displaced downward in the body into the capacitance areas of the abdomen and extremities, and away from the brain. The human body is restrained in place in an ejection seat as the $+\mathrm{Gz}$ stress is applied. Both the body and the blood are pushed downward. In relation to the stress, however, the body is held essentially stationary, and since the blood vessels are distensible, the blood pools in the most dependent areas of the body. The ability of the heart to continue to supply blood to the brain is easily compromised around $+5 \mathrm{Gz}$ in an unprotected, relaxed human.

The exact level of $+\mathrm{Gz}$ stress that results in loss of adequate blood flow to the brain is dependent on many factors. Modern fighter aircraft can routinely achieve and sustain levels of $+9 \mathrm{Gz}$, which places most humans at risk for +Gz-induced loss of consciousness (G-LOC). It is not only the high levels of $+\mathrm{Gz}$ stress that are important; the very rapid application and sustained level of the $+\mathrm{Gz}$ stress must 
also be considered. Again, modern fighter aircraft can produce $+9 \mathrm{Gz}$ in one second ( $9 \mathrm{G} / \mathrm{s}$ onset rate) and maintain that level for sustained time periods (greater than 5 seconds). The magnitude of such stress can be understood by realizing that a 200 pound man at $+1 \mathrm{Gz}$, the everyday gravitational stress experienced by earthbound humans, will be transformed in one second or less at $+9 \mathrm{Gz}$ to an 1800 pound man in a fighter aircraft. The unassisted heart and vascular systems are unable to compensate for such stress, and ischemia results in the cephalic nervous system causing loss of consciousness. Research using a ground-based human centrifuge stimulates in-flight $+\mathrm{Gz}$ stress and is conducted to develop life support equipment and techniques to protect fighter aircrew from the adverse affects of $+\mathrm{Gz}$ stress. In the course of such research many loss of consciousness episodes result.

The results to be discussed represent data collected from 16 years of acceleration research experience and nearly 1000 episodes of GLOC that occurred in fighter aircraft and during centrifuge $+\mathrm{Gz}$ exposure. The individuals included experimental research subjects, fighter aircrew, and students in various aviation medicine courses. The average age of the individuals was approximately 32 years. All were healthy and asymptomatic, having successfully completed military flying physical examinations. The centrifuge G-LOC episodes were all recorded on videotape for subsequent analysis, as described elsewhere (Whinnery, 1989b). Results of the physiologic analysis of the centrifuge G-LOC episodes have been reported (Whinnery and Whinnery, 1990). The sequence of events for a typical G-LOC episode is shown schematically in Figure 1.

When rapid onset $+\mathrm{Gz}$ stress was applied to a sustained level well above tolerance, there was an approximately 6-second period (functional buffer period) during which normal neurologic function persisted. At the end of this period, consciousness was lost and the $+\mathrm{Gz}$ stress was reduced back to resting conditions. The length of the unconsciousness episodes averaged 12 seconds ( \pm 5 seconds), with a range of 2 to 38 seconds. The estimated average length of time blood flow to the central nervous system was altered to a level causing loss and recovery of consciousness was approximately 15 to 20 seconds. Myoclonic convulsive activity was observed in association with 70 percent of the G-LOC episodes. The myoclonic activity began on the average 7.7 seconds after the onset of unconsciousness and lasted 3.9 seconds. The myoclonic activity was observed to stop coincident with the return of consciousness. Upon recovery of consciousness 
Figure 1. Kinetic quantification of the sequence of events associated with a typical +Gz-induced loss of consciousness (G-LOC) episode resulting from a rapid-onset and moderate-offset $+\mathbf{G z}$ profile.

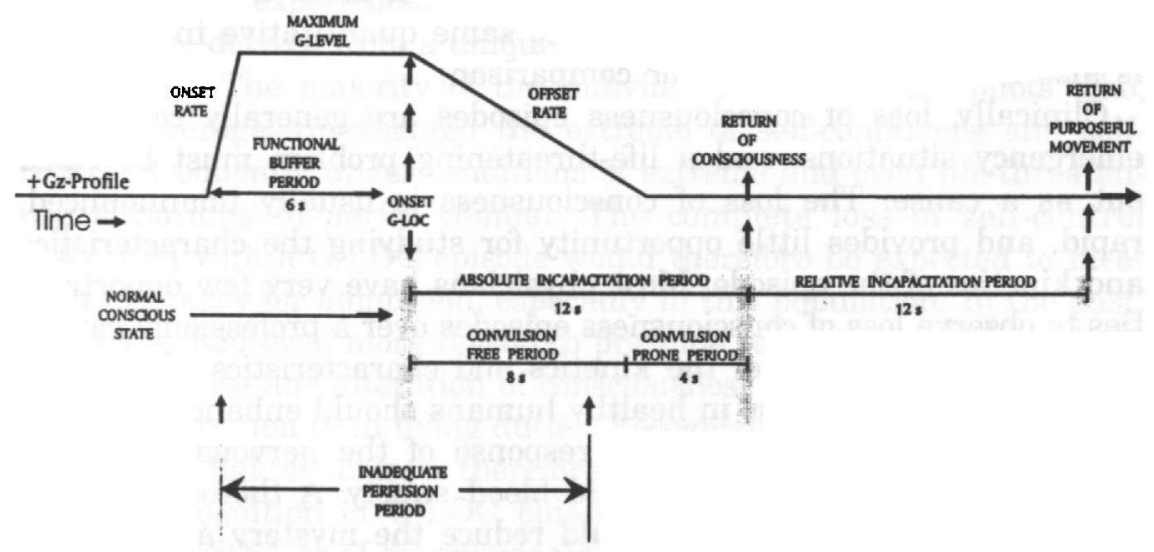

there was a period of relative incapacitation that lasted on the average about 12 seconds. This was a period in which there was confusion and disorientation. The end of this period was ascertained by the return of the ability to make purposeful movement, that is, regaining aircraft control or performing other specific tasks. Individuals who lost consciousness were interviewed in the centrifuge on videotape immediately following the G-LOC episode. Immediately after leaving the centrifuge gondola all individuals were required to complete a questionnaire describing the $+\mathrm{Gz}$ exposure and all experiences associated with the G-LOC episode. Many of the individuals were also interviewed at several follow-up times up to several months subsequent to the G-LOC episodes.

\section{Results}

After reviewing this large number of G-LOC episodes in completely healthy humans, a pattern of experiences emerged from this specific type of ischemic insult to the nervous system. It is not the purpose of this report to describe exhaustively all aspects of G-LOC episodes. The intent is to present findings from our aeromedical research that may be pertinent to those whose research is aimed more directly at understanding NDEs and associated phenomena. Only the general 
aspects of our G-LOC experiences will be reviewed in a qualitative manner, with a limited number of specific examples provided to illustrate major points of importance. It is important to provide as much quantitative information as possible concerning the frequency of occurrence of the symptoms associated with these G-LOC episodes (Whinnery and Whinnery, 1990). The same quantitative information is also needed from NDEs for comparison.

Clinically, loss of consciousness episodes are generally considered emergency situations, and a life-threatening problem must be ruled out as a cause. The loss of consciousness is usually unannounced, rapid, and provides little opportunity for studying the characteristics and kinetics of the episode. Most physicians have very few opportunities to observe loss of consciousness episodes over a professional career. A complete description of the kinetics and characteristics of loss and recovery of consciousness in healthy humans should enhance the overall understanding of the normal response of the nervous system to transient alterations of the normal blood supply. A thorough understanding of the phenomenon should reduce the mystery and misconceptions associated with loss of consciousness and near-death episodes.

\section{Recognition of G-LOC}

The experiences of the subjects during the G-LOC episodes were collected through individual reports, personal interviews, and questionnaires. Considering all G-LOC episodes observed, the immediate interview following the episode revealed that approximately 50 percent of the individuals did not immediately recognize that they suffered a loss of consciousness episode. Many were shocked to observe their unrecognized G-LOC episodes on videotape review after exiting the centrifuge. This problem with recognition of the G-LOC episode is complicated by the acknowledged fact that many of the subjects experienced a psychologic urge to suppress or deny that they had been unconscious. The individuals found it difficult to describe this urge to try to hide their loss of consciousness, but indicated that it frequently resulted in their keeping quiet while hoping for someone to provide them with adequate information to fill in their G-LOC void. This existed in spite of their knowledge that the entire exposure was being captured on videotape. Many subjects automatically, and essentially uncontrollably, attempted to fill in their void by confabulation. The ability to recognize a G-LOC episode is definitely related 
to the severity of the neurologic insult, as measured by the duration of the absolute incapacitation period. The longer the absolute incapacitation period, the more likely one is to recognize that G-LOC had occurred (Whinnery and Whinnery, 1990).

The G-LOC experiences I had the opportunity to observe and investigate were derived from a unique segment of the population of healthy individuals. The majority of the individuals were fighter pilots who, on the average, represented the extreme of self-confidence and selfcontrol, frequently under conditions of extreme and even life-threatening situations in aerial combat. The complete loss of self-control associated with a G-LOC episode would therefore be expected to meet with hesitancy for admission, especially in this population. In the past, pilots also had even more reason to preclude reporting in-flight G-LOC episodes, since any alteration of consciousness was, by regulation, reason for restriction from flying duties. Recognizing and reporting G-LOC episodes in fighter aircrew therefore may have at least 3 components: lack of recognition of G-LOC episode, psychophysiologic suppression associated with loss of consciousness, and reluctance to report a G-LOC episode even if recognized. All three of these reasons would apply in one way or the other to the NDE. It is only recently that reporting the NDE has been encouraged; keeping it a secret however, is more complicated than simply fear of being considered mentally unstable (Dlin, Stern, and Poliakoff, 1974; Druss and Kornfeld, 1967). To quote a written communication from a naval aviator:

Only after reading of your work have I come to realize I'd G-LOC'd at least 3 times. I always felt foolish after them and told myself to pay more attention, work harder, etc. I only really remember waking up once while in a F-4 [aircraft] during an ACM [air combat maneuvering] hop.

It is possible to classify the G-LOC episodes into four types, according to the pilot's recognition of loss of consciousness and the presence of dreamlets. For the most transient episodes there is frequently no recognition at all. I classify this as absolute lack of recognition of the loss of consciousness. If handled appropriately during centrifuge exposure, individuals can be encouraged to continue on as though nothing had happened. It is only when individuals reviewed their own videotapes that they understood, with amazement, that they had suffered G-LOC episodes. The second type of experience is associated with knowing that something was unusual, but being unsure exactly what it was. I classify this as relative lack of recognition of having 
been unconscious. In general, as the ischemic insult increases, the likelihood of recognition of loss of consciousness increases. Both the third and fourth types of experiences are recognized. The third type of experience is associated with a clear knowledge of the loss of consciousness; however, these individuals do not have a dreamlet, or at least are unable to remember if they had a dreamlet or, if they did, exactly what the dreamlet content was. This type of G-LOC episode is recognized, but no report of a dreamlet can be described. They may be able to describe many, if not all, of the other aspects of a G-LOC episode. The fourth type of experience is one in which the individual has a memorable dreamlet and is able to remember and describe all the major aspects of the experience. This classification scheme is a reflection of the relationship between the magnitude of the ischemic insult and the resulting symptoms that are produced.

\section{Visual Symptoms}

The most common symptoms associated with exposure to $+\mathrm{Gz}$ stress are related to alterations of vision. Because of the unique characteristics of the eye in comparison to the remainder of the nervous system, visual symptoms occur at lower $+\mathrm{Gz}$ levels (and higher blood supply pressures) than symptoms for the rest of the nervous system. To understand this physiologic phenomenon, it must be recognized that the eye has an increased pressure compared to the remainder of the nervous system. This intraocular pressure is roughly $20 \mathrm{~mm}$ $\mathrm{Hg}$. As the $+\mathrm{Gz}$ stress increases, perfusion to the retina is compromised at lower $+\mathrm{Gz}$ levels than the other surrounding nervous tissue. As the perfusion pressure to the eye falls during exposure to $+\mathrm{Gz}$ stress, the most distal circulation within the retina is compromised first, producing loss of peripheral vision. As the $+\mathrm{Gz}$ level increases further, the visual field contracts to produce what is known as tunnel vision. This results when perfusion pressure is enough to supply only the small area where the central retinal artery enters the eye. Finally, when the perfusion pressure is so low that no blood flow to the retina occurs, blackout, or complete loss of vision, results. Blackout is not loss of consciousness. If the rate of onset of $+\mathrm{Gz}$ is gradual, then the progression of visual symptoms from "grayout" (loss of peripheral vision) to tunnel vision to blackout can be observed. These visual symptoms precede loss of consciousness. If the rate of onset of $+\mathrm{Gz}$ is very rapid, then the progression of symptoms may not be 
observed or, if observed, not remembered. The most frequent sequence of visual symptoms is such that the last observation prior to loss of consciousness is some degree of tunnel vision.

It is difficult to assess the visual symptoms that occur during the transition from unconsciousness to recovery of consciousness, since the human is not fully conscious while these symptoms are occurring. From a perfusion mechanism standpoint it is plausible that the reverse sequence of symptoms occurs. This means that many of the neurologic faculties required for full consciousness would be well on their way to recovery as normal vision is being restored, based on the increased intraocular pressure. Specifically, this sequence would include the presence of tunnel vision coincident with the earliest recovery of conscious function. The kinetics of the visual recovery and recovery of the remainder of neurologic function is consistent with tunnel vision occurring at the same time we believe the dreamlets are occurring, as consciousness is returning near the end of the absolute incapacitation period. Just as we have observed other physical and physiologic activities, such as myoclonic jerking of the extremities, being incorporated into the dreamlet, we would predict that the coexistent visual symptoms would likewise be incorporated into many of the G-LOC dreamlets. The incorporation of tunnel vision into the dreamlet could logically be interpreted as coming out of the darkness of a tunnel or perhaps bright sunlight. Even without this coincident sequence during recovery, the last observation prior to loss of consciousness is usually tunnel vision, and therefore its frequent incorporation into the G-LOC experience dreamlet would not be an unreasonable expectation. In summary, tunnel vision is sequentially the last visual input to the brain as consciousness is degraded, and a visual input to the brain just prior to the return of full consciousness.

The visual symptoms and physiologic changes that result from exposure to $+\mathrm{Gz}$ stress are not unique to $+\mathrm{Gz}$ stress. Any process that results in reduced perfusion to the nervous system can produce similar symptoms. These other processes, such as cardiac asystole, may also be responsible for similar visual symptoms that can contribute to a loss of consciousness experience.

\section{Myoclonic Convulsions}

From research on the kinetics of G-LOC it became clear that the myoclonic jerking occurred at a specific time during the G-LOC epi- 
sode. As shown in Figure 1, for the type of ischemic insult +Gz stress generally delivers to the nervous system, the myoclonic jerks occur at the end of the absolute incapacitation period, they last approximately four seconds, and they end essentially coincident with the return of consciousness. This sequence results in the myoclonic jerking occurring as the individual is regaining consciousness. The apparent inclusion of myoclonic jerking into the dreamlet is one of the prime reasons that we consider the dream period to be near the end of the absolute incapacitation period. Dreamlets in which individuals stated they were riding on "bumper cars" at an amusement park were reported when they rhythmically jerked their heads against the headrest of the seat. Rhythmic jerks of the arms were reported as one individual experienced a dream about fishing and pulling back on his fishing rod several times to set the hook. Floundering around in the water while being about to drown was reported in association with the rhythmic extremity jerking of the myoclonic convulsions. The frequent but not universal incorporation of such coexisting physiologic activity into the dreamlet has been previously reported. Daniel Dennett (1993) gave an example of incorporating coexisting physiologic stimuli into the dream content: in his own dream he heard a lamb bleating rhythmically, which then coalesced perfectly in cadence with his telephone ringing as he awoke.

\section{Memory}

Memory is certainly compromised in association with a G-LOC episode. Just as with other neurologic functions, the exact kinetic relationship of memory compromise is dependent on the time course and magnitude of the ischemia along with the neurologic structures that are affected. Memory must return prior to the end of the absolute incapacitation period, since the dreamlet, myoclonic convulsions, and paralysis frequently can be remembered. The exact kinetics of neurologic substrate recovery, however, remain unknown.

The induction of unconsciousness is closely associated with compromise of the memory processes. It is difficult, however, to assess the exact moment when memory is lost. Following the experimental determination of G-LOC kinetics and establishment of a theoretical mechanisms of G-LOC (Whinnery, 1989c) I predicted that if the ischemic insult was carefully titrated, the symptoms observed during 
recovery should also be produced during the induction of unconsciousness (Cammarota, 1992).

Centrifuge experiments were subsequently conducted that involved deliberate induction of unconsciousness using prolonged, cyclic $+\mathrm{Gz}$ stress in association with a complex simulated aerial combat maneuvering tracking task. These experiments confirmed that myoclonic convulsive activity could be induced prior to G-LOC and also that memory compromise could be induced prior to G-LOC. Utilizing a complex flight tracking task, highly trained individuals were asked to report the last point they remembered in the tracking task before losing consciousness. This sequence in the tracking task could then be accurately determined. It was also possible to determine coincidentally at what exact point during the tracking task that tracking ability - and consciousness-was lost. The point of last memory generally preceded the loss of tracking ability, indicating that memory was compromised prior to loss of consciousness. The validity of this G-LOC model was not only enhanced by the ability to predict such phenomenon correctly; it indicated that memory could well be compromised for a very short period of time prior to G-LOC. Observation of such memory compromise requires rather sophisticated measurement techniques. If there is a very short but finite period of memory compromise prior to G-LOC, it might also be possible for an event to be captured by the senses yet not recorded accurately in memory as G-LOC occurs. As such, it would be possible for sensory input to occur, that is, be observed by the senses, but not remembered. Our aerial combat tracking task data suggest that this does occur (Cammarota, 1992).

During recovery of consciousness it is evident that mnestic processes return prior to consciousness. At least some of the evidence supporting this contention is based on the fact that the dreamlets and myoclonic convulsions occur prior to the end of the absolute incapacitation period and the return of consciousness, and are indeed remembered. Therefore, there are processes within the nervous system that become functional prior to the return of consciousness. Memory is one of those processes that returns prior to the return of consciousness. Captured within functional memory are the physiologic inputs that transpire prior to the return of consciousness. The fact that mnestic processes in general return also provides the compendium of the individuals' prior thoughts, experiences, and ideas that can be incorporated into the experience. It is within this milieu of existing physiologic sensory and psychologic inputs, along with the 
information contained in memory, that the G-LOC experiences seem to be formulated, remembered, and subsequently reported.

\section{Psychophysiologic Symptoms}

I have already described the major characteristics of G-LOC experiences, which include the incapacitation, myoclonic convulsions, alterations of the memory processes, and the visual symptoms. I will defer the description of dreamlets until last, since they are generally associated with the summation of all psychophysiologic events that transpire during the G-LOC episode. The additional physiologic symptoms of G-LOC reported include tingling distally in the extremities and around the mouth, post-G-LOC mild confusion and disorientation, a feeling of awakening from sleep, and peri-awakening paralysis. Individuals also have psychologic alterations in association with the G-LOC episodes, including embarrassment, euphoria, dissociation, anxiety, fear, antagonism, inability to concentrate, and a "give-up" or submission attitude. The embarrassment was present even when the centrifuge acceleration exposure was part of a research study specifically designed to result in unconsciousness, the unconsciousness being a successful experimental outcome. A sense of floating is one of the most frequently reported symptoms associated with G-LOC experiences. It is commonly associated with autoscopy, automatic movement, paralysis, a sense of dissociation, and being pleasurable enough to result in the individual not wanting to be disturbed. Overall, the majority of individuals consider the G-LOC experience not only pleasurable, but euphoric. Much less frequently, on the other hand, an occasional individual does find the experience to be disagreeable and frustrating.

Many of these psychologic alterations persist for several hours following the G-LOC episode, with the individuals indicating that they feel normal only following a subsequent period of sleep. This is particularly true for the feelings of detachment, dissociation, and the inability to concentrate. Although not a common occurrence, out-ofbody experiences (OBEs) are reported in association with G-LOC episodes. These OBEs have been associated with longer G-LOC episodes and multiple, closely-spaced G-LOC episodes, as many as five within a 15-minute period (Whinnery and Jones, 1987). This may indicate that OBEs are more likely to occur with increasingly severe ischemic insults to the nervous system. One associated $\mathrm{OBE}$, for example, oc- 
curred following multiple G-LOC episodes. The individual walking down a hallway immediately after a centrifuge exposure became aware not only that he was walking down the hallway, but also that he was above and behind himself, watching his own body walking. The duality persisted for approximately three minutes before the experience ended with what was described as a reintegration process with his body.

The above symptoms might be predicted to result from $+\mathrm{Gz}$ stress causing G-LOC, as illustrated in Figure 2. Loss of sensory input to the nervous system essentially produces a de-afferentiation. Loss of motor output eliminates the effective link of the nervous system with the remainder of the organism, or de-efferentiation, the net result being a perception of nervous system detachment or dissociation and the sensation of floating or being out of, and not connected to, the body.

Figure 2. Kinetic sequence of the loss and recovery of consciousness effectively resulting in the isolation of the nervous system from its environment, including the rest of the body.

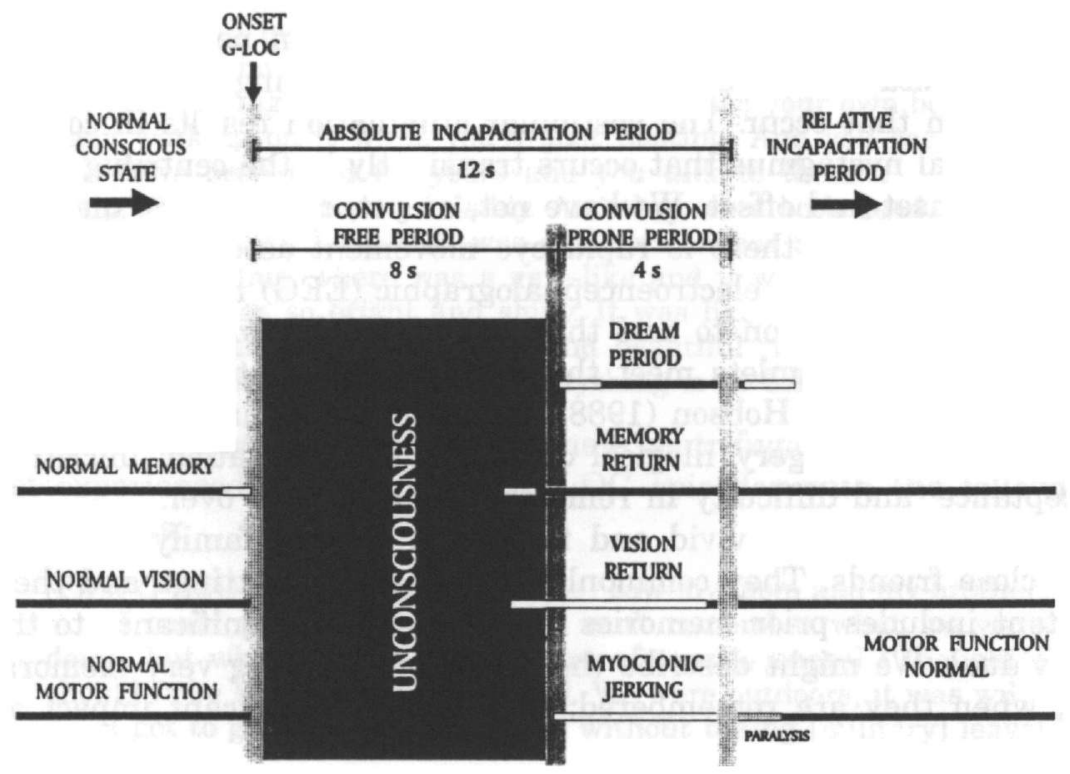




\section{Dreamlets}

The characteristics of G-LOC episodes in which dreamlets occur have been reported elsewhere (Forster and Whinnery, 1988; Whinnery and Whinnery, 1990). It is clear that dreamlets are much more likely to occur and be memorable as the severity of the ischemic insult increases; there is a strong correlation between memorable dreamlets during G-LOC and the magnitude of the ischemia (Whinnery and Whinnery, 1990). The longer the absolute incapacitation period, the more likely it is that a dreamlet will be reported. Overall, our results suggest that about 35 to 40 percent of our G-LOC episodes have dreamlets, or closely related thought processes that occur and are reportable. We have chosen to refer to these as dreamlets since the individuals describe them as indistinguishable from the dreams they experience during regular sleep, the only difference being the short duration of G-LOC dreamlets in comparison to sleepassociated dreams. It should be noted that these G-LOC experiments allow a dreamlet to be isolated to a specific 12 -second or shorter period of time.

An immediate question that arises regarding G-LOC dreamlets is whether or not they are associated with rapid eye movements. This is an important question because the answer would enhance our ability to link our results with sleep dream research. The question is not easily resolvable however, not only because of the very short duration of the G-LOC episode, of which the dream period is only a part, but because of the concurrent myoclonic jerking and vestibular stimulation that occur. The vestibular stimulation results in coriolis and vertical nystagmus that occurs transiently in the centrifuge during $+\mathrm{Gz}$ onset and offset. We have not, as yet, resolved the question of whether or not there is rapid eye movement associated with GLOC dreamlets. The electroencephalographic (EEG) response during G-LOC is a transition to and then out of a delta wave pattern. In any case, our dreamlets meet the characteristic features of dreams described by Allan Hobson (1988), including emotional intensity, detailed sensory imagery, illogical content and organization, uncritical acceptance, and difficulty in remembering once it is over.

The dreamlets are vivid and frequently include family members and close friends. They commonly have beautiful settings and their content includes prior memories and thoughts of significance to the individual. We might describe the dreamlets as being very memorable, when they are remembered; they have a significant impact on 
individuals who experience them, and remain crystal clear for years after they occur. There is a very strong urge in most individuals to try to understand and explain what they experienced.

\section{The G-LOC Experience}

The major characteristics of G-LOC experiences that are shared in common with NDEs include tunnel vision and bright lights, floating sensations, automatic movement, autoscopy, out-of-body experiences, not wanting to be disturbed, paralysis, vivid dreamlets of beautiful places, pleasurable sensations, psychological alterations of euphoria and dissociation, inclusion of friends and family, inclusion of prior memories and thoughts, the experience being very memorable (when it can be remembered), confabulation, and a strong urge to understand the experience. The similarities between NDEs reported by patients and the G-LOC episodes reported by healthy individuals may be compared in a few examples taken from G-LOC and NDE descriptions.

NDE \#1. A 39-year-old housewife described the following neardeath experience resulting from a cardiac arrest (Sabom and Kreutziger, 1977, p. 650):

I knew I was dying because the pain was so bad. . . it was like a bullet hitting my heart. ... I called the nurse and by then she couldn't find no respiration or nothing. . . . And you could see yourself just floating up in the air and you could see your own body and them working on it while you're just floating. And you see people who have been dead for years and you talk to them. . . . I love them, like my mother and daddy. And they were both dead. In fact she had her own baby that was still-born in her arms ... it was like a borderline. There was a gate-like and it was real beautiful . .. the sun was so bright and shiny. It was like another world ... it's white with clouds and grass and beautiful trees . . . like this but a beautiful sunny day, but everything is so bright and clean.

G-LOC subject \#1. A 20-year-old male centrifuge experimental subject experienced two successive G-LOC episodes with the following descriptions:

[First G-LOC episode] I was home . . saw my mom and my brother. ... I could not see myself. . . I I can't remember what we were doing, but when I came back [return of consciousness] I thought I shouldn't be here [in the centrifuge]. We were outdoors; it was wild! ... I got to go home [by dreaming] without taking [military] leave! 
He knew he was in his home in Michigan, and this dreamlet was vivid and in color.

[Second G-LOC episode] There was a sunset. I cannot remember where. The sun was orange-red; an October sunset . . . . Maybe it was in Michigan.

He considered this experience very intense and pleasant.

G-LOC subject \#2. A 31-year-old male centrifuge experimental subject who suffered a G-LOC episode described the following experience:

I was floating in a blue ocean, on my back . . kind of asleep but not asleep. I knew the sun was up ... like someone was trying to wake me up. Finally, I woke up and I was on the centrifuge! I did not want to wake up. ... I could see myself on the water and also look at the sun; the sky was very blue, the sun very yellow.

The subject stated that he enjoyed the experience, especially the sensation of floating.

$N D E$ \#2. A typical response, from a patient-physician who had a near-death experience resulting from ventricular fibrillation associated with a myocardial infarction, included the following (Anonymous, 1969, p. 263):

... I could not phonate. The words just would not come out. I decided to feel for his [the doctor's] arm (because I was still unable to see) and was distressed to find I could not move either hand.

A similar incident was reported by Barney Dlin, Andrew Stern, and Steven Poliakoff $(1974$, p. 63):

One patient recalled thinking 'I'm trying to signal you that I'm alive," and feeling a tremendous sense of desperation because "my lips wouldn't move . . . I couldn't even move my hand."

G-LOC subject \#3. This sense of paralysis and associated frustration is extremely common in G-LOC episodes. A typical example from a centrifuge subject is as follows:

I was in the grocery store going down one of the aisles. I was ... being propelled by something like a magic carpet, although I could not make movements. I wanted to reach out and get a carton of ice cream but could not move my arm or even my eyes to look for it. It was intensely frustrating to hear the warning horn and not be able to get my arm down to turn the darn thing off.

The sense of paralysis was extremely frustrating, especially as he found he could hear a G-LOC warning tone in the centrifuge and 
could not move to turn it off initially as consciousness returned. It was apparent that sensory function, memory, vision, and hearing returned before return of motor function and the ability to move the upper extremities.

Both G-LOC and the NDE are usually very significant events that can result in subsequent behavior modification. It has been shown that in subsequent G-LOC episodes following the first one, the period of relative incapacitation (confusion/disorientation) is reduced. This demonstrated ability to recognize that G-LOC has occurred and then recover more quickly has prompted us to recommend that all fighter aircrew undergo "G-LOC training" as an enhanced flight safety measure (Whinnery and Burton, 1987).

The significance of an in-flight G-LOC episode is illustrated by an incident that occurred to a distinguished fighter pilot in an F-86 aircraft. The pilot had an in-flight G-LOC episode with an associated dreamlet about flying his fighter aircraft. The pilot recovered his aircraft without incident and had not previously told anyone about the G-LOC episode over the subsequent 20 years. The pilot related that since his in-flight G-LOC episode, he had trained himself to wake up whenever he had a normal sleep dream that involved flying a fighter aircraft. The reason for his actions was that he could never be sure whether he was asleep and dreaming about flying or whether he was actually flying, suffering a G-LOC episode, and having a dreamlet about flying.

\section{Discussion}

The resulting psychophysiologic symptoms and alterations produced by transient $+\mathrm{Gz}$-induced ischemia and hypoxia of the cephalic nervous system have been collectively described as the G-LOC syndrome (Whinnery, 1990). A lot happens in a very short time over the course of the loss and recovery of consciousness. On the average, this sequence of degradation and recovery of normal neurologic function takes from 12 to 24 seconds. The experimental evidence reveals a consistent pattern of both physiologic events and psychologic experiences that occur in the course of ischemic challenge to the nervous system. It is logical to assume that the psychologic experiences reported result from the overall neurologic substrate configurations that are sequentially produced. The overall neurologic configurations result from fully functional areas, functionally compromised areas as 
blood supply to the cephalic nervous system is modified, and the interactions between combinations of functional and nonfunctional neurologic substrate configurations.

The overall perceived experience of a G-LOC episode represents the complex integration of all the psychophysiologic inputs and memory existing within the nervous system during various stages of functional neurologic capability. When consciousness has been lost and then recovered, the nervous system is faced with logically integrating all its prior and current activities-sensory input, memory, analysis, and responsive output-into a rational experience that "makes sense." In trying to develop such an overall rational experience, the nervous system has to work with the information that it gained from a minimally functional system through various stages of neurologic function, up to and including a fully functional system. It has to accomplish this essentially instantaneously. Such a remarkable feat results in the experience that an individual reports when asked: "What do you remember?" An abrupt alteration such as loss of consciousness would likely leave whatever was occurring in the nervous system open-ended and not disposed of in the usual manner. It is not surprising that some loose ends may be tied together that are not quite in the usually correct sequence for being tied together. Perhaps the nervous system indeed recognizes this, which would explain why many subjects who have G-LOC episodes report they remain feeling dissociated until a period of sleep occurs. Sleep could potentially serve as a period that provides the nervous system an opportunity to consolidate more appropriately the loose ends resulting from an abrupt interruption of consciousness. For these reasons, pilots who have a G-LOC episode are usually restricted from flying until a sleep period has transpired.

For a G-LOC episode, it is important to develop a composite list of all the co-existing sensory input present during the average 12second period of altered consciousness. This sensory input represents all of the new information that might be made available in the nervous system, combined with the already existing information within short- and long-term memory. Memory function itself is also in various stages of functional compromise as consciousness is lost and regained, and therefore contributes to the experiences in a piecemeal fashion. This complex sum of information is what is available within the nervous system during a G-LOC episode. A complication arises because some of the sensory input may be received when the nervous system is only partially functional and therefore may be processed 
in a nonstandard manner. Some of the sensory input that must be considered in association with a G-LOC episode in the centrifuge includes vestibular stimulation, such as a tumbling sensation known as coriolis; myoclonic convulsive movements; auditory stimulation, such as the G-LOC warning tone; visual stimulation, such as the G-LOC warning light; tunnel vision; paralysis, or loss of motor control; and transient loss of sensory ability.

Although some of these stimuli may also occur in NDEs and "sudden death" episodes, others do not. Vestibular stimulation, for instance, is unlikely to be associated with anything except a G-LOC episode. Myoclonic convulsive activity may or may not be as frequently associated with NDEs or "sudden death" episodes. We have observed vestibular manifestations and myoclonic convulsive movement manifestations in G-LOC dreamlets. Such unique events would be expected to be observed in G-LOC episodes, but rarely if ever in NDEs or "sudden death" episodes, unless the necessary physiologic stimulation occurred. On the other hand, most of the other sensory events are present in both, and would be predicted to be a frequent part of both G-LOC and NDEs. This includes such events as tunnel vision, paralysis, and auditory and visual environmental sensations. A complete kinetic categorization of such sensory events for both NDEs and loss of consciousness episodes are key aspects of understanding both phenomena.

The main point of the current discussion is that we observe G-LOC experiences in which dreamlets occur whose content frequently reflects the inclusion of coincident physiological stimuli. The best examples of this are the occurrence of myoclonic convulsions, paralysis, and tunnel vision that are incorporated into the dreamlets. We would therefore postulate that many of the same or similar occurrences would be a part of NDEs and "sudden death" episodes. The insult to the nervous system in NDEs is very likely much more severe, and that difference should be reflected in the reported events. It would appear that many, but not necessarily all, of the characteristics of NDEs are consistent with what happens with a loss of consciousness episode. All the events being received and processed in the functional nervous system must ultimately be incorporated into memory in order for them to become included in the NDE or G-LOC experience. Memory must be functional in order for the experience to be memorable and subsequently reported. It should be recognized that NDErs who fail to recover completely may not be able to report their experiences or, for that matter, even respond. Even the individuals who 
are able to report the NDE may, in fact, have permanent anatomic and physiologic alterations or damage that may influence the NDE. These NDEs would be expected to be significantly different from GLOC experiences.

\section{A Protective Mechanism}

Loss of consciousness is considered only a symptom in clinical medicine, and because of that it has not been systematically studied. Clinical medicine has little motivation to study in depth something that can be prevented by correcting the underlying problem that causes it; no one dies directly from loss of consciousness. In addition to its clinical classification as a symptom, there remains considerable fear about transient loss of consciousness being a dangerous event. Therefore, there have been considerable barriers to conducting experiments involving ischemia or hypoxia in completely healthy humans. At least part of the concern over loss of consciousness being potentially pathological comes from its automatic clinical association as a symptom with life-threatening primary problems such as cardiac arrest. The loss of consciousness is usually what we observe in a cardiac arrest, not the actual asystole or ventricular fibrillation. The real concern, however, is not the loss of consciousness, but rather the cardiac arrest (ischemia) persisting long enough for nervous system pathology to occur.

In contrast, loss of consciousness is considered a primary problem in fighter aviation medicine. The unique risk/benefit ratio in fighter aviation medicine provides the opportunity to investigate the G-LOC problem. For many years, even in aviation medicine, the clinical concern associating loss of consciousness with life-threatening disease processes made G-LOC experiments in healthy humans difficult. Visual symptoms such as tunnel vision and blackout were used as experimental endpoints so that G-LOC could be avoided.

Conducting G-LOC experimentation safely rests primarily on the understanding that G-LOC is a protective mechanism (Whinnery, 1989a, 1991). When the nervous system is challenged with +Gz-induced ischemia, the processes associated with G-LOC are initiated to prevent pathologic insult to the nervous system. Evolutionary development in the environment of the earth's gravitation $(+1 \mathrm{Gz})$ would logically provide such a mechanism to preserve nervous system integrity to the greatest extent possible. When nervous system is- 
chemia occurs as a result of $+\mathrm{Gz}$ exposure, a mechanism that not only reduces the energy expenditure requirements of the nervous system but also removes the upright organism from the $+\mathrm{Gz}$ stress field would be extremely advantageous for survival. This is exactly what G-LOC does. Nervous system energy is conserved by reducing the expenditures required by conscious functioning, and the body is placed in a horizontal position, with heart and brain at the same level in the $+\mathrm{Gz}$ field. Loss of consciousness is a normal process within the normal nervous system. A critical balance exists between consciousness, the neurologic state that is key for survival of the organism, and unconsciousness, the neurologic state in which the nervous system is optimally protected against $+\mathrm{Gz}$-induced ischemic insult. A complete description of the entire G-LOC protective mechanism has been developed with the major sequence of events shown in Figure 3.

With respect to the current discussion concerning the overall GLOC experience, the dreamlets and psychologic alterations are important protective mechanism components. Since G-LOC episodes are difficult to recognize and remember, it is key for there to be mechanisms that enhance the ability of the organism to recognize an event that threatens survival. The dreamlet and psychologic alterations serve as a mechanism to enhance the recognition of such an event. Recognition thereby serves to allow the organism to avoid the same situation or environment in the future. It would be logical that the same mechanism serves similar purposes for NDEs. Situational avoidance and behavior modification would be expected if threats to survival are recognized by the organism.

\section{Neurologic States}

Treatment of the nervous system by classical thermodynamics results in the establishment of neurologic states based on energy relationships (Whinnery, in press). Consciousness and unconsciousness represent different states of the nervous system. Relative to $+\mathrm{Gz}$ stress affecting the otherwise normal nervous system, the neurologic state is dependent on the existing neurologic energy. The transitions between consciousness and unconsciousness that result from manipulation of $+\mathrm{Gz}$ stress are caused by energy (blood supply) alterations. Between these two states there is the subconscious state, which corresponds to a partially functioning nervous system. Each of these 
Figure 3. Schematic flow diagram of the postulated psychophysiologic protective mechanism sequence that underlies +Gz-induced loss of consciousness (G-LOC). The nervous system is housed within the body, which serves to provide anatomic protection.

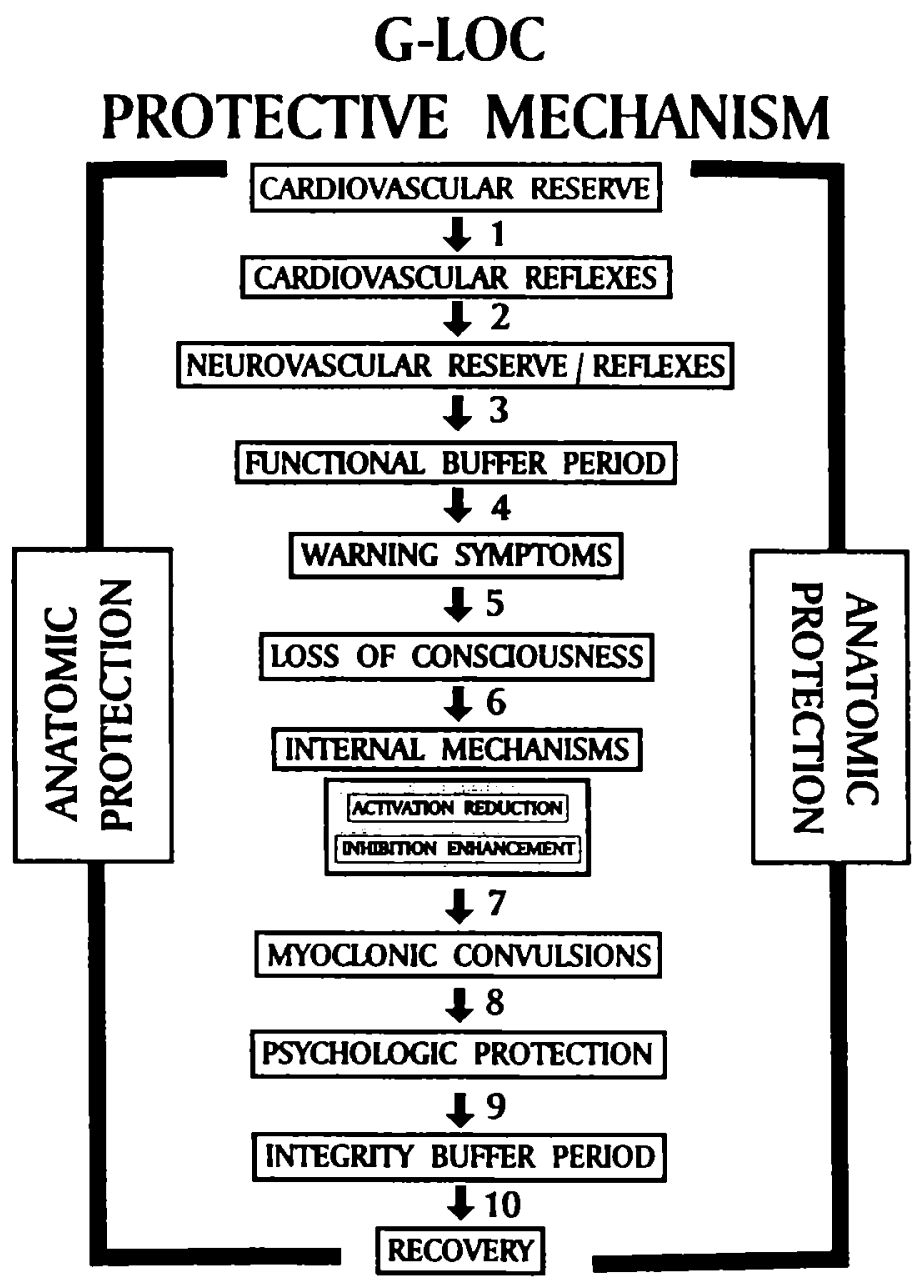

neurologic states-consciousness, subconsciousness, and unconsciousness-represents a range of neurologic energy distributed over a spe- 
cific nervous system structure. It is necessary to define at least one additional neurologic state, a state that corresponds to a critical range of reduced energy, from which, by definition, it is impossible to regain a neurologic state above unconsciousness. This neurologic state is death.

The relation of these neurologic states is illustrated in Figure 4. The neurologic state of consciousness is a high energy state as compared to the lowest energy state of death. Loss of consciousness induced by $+\mathrm{Gz}$ stress results from decreased energy and results in transitions through the subconscious state to unconsciousness. Recovery of consciousness requires the restoration of energy to the nervous system and transition from unconsciousness through the subconscious state back to the normal conscious state. When the insult mechanisms are similar for G-LOC and NDEs-for instance, cardiac arrest and G-LOC-the general difference between the two experiences becomes a difference in the kinetics of the processes, nervous system ischemia being more frequently prolonged in the NDE. These neurologic energy-state relationships provide a theoretical framework for understanding the G-LOC syndrome and NDEs.

Figure 4. Comparison of the theoretical energy-dependent processes associated with $+\mathbf{G z}$-induced loss of consciousness (G-LOC) episodes and with near-death experiences (NDEs). The resulting experiences reported upon recovery are dependent on the depth and duration of time within the states of the nervous system.

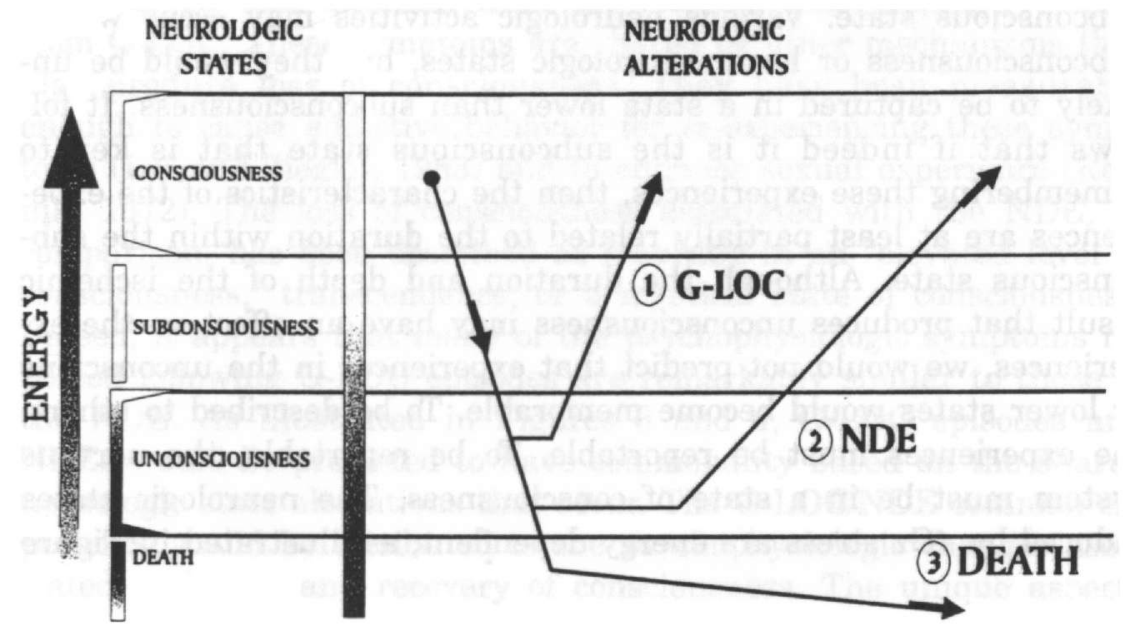




\section{Experiences Involving Unconsciousness}

Both NDEs and G-LOC experiences commonly involve a transient sojourn into the unconscious state. Both also involve a return to the conscious state with the ability to recall certain activities that occurred sometime during loss of consciousness, the transitions through subconsciousness and unconsciousness, and/or the return of consciousness. The differences between these experiences in a normal nervous system are related to the magnitude, duration, and distribution of energy deprivation within the nervous system. As previously described, for the activities to be remembered, various areas of the nervous system, including those underlying memory, must be functional. For the experiences to be reported, the nervous system must ultimately return to a state of consciousness that is considered essentially normal. Although changes may have occurred, including pathologic ones, they cannot be so extensive or critically located to prevent establishment of the state of consciousness within the normal range, if the experience is to be accurately reported. Evidence from G-LOC experiences reveals that memory is compromised when an individual is unconscious. Evidence also reveals that memory becomes functional before the state of consciousness is restored. Memory is at least partially functional within the subconscious state that exists during transitions between consciousness and unconsciousness.

We would therefore predict that the content of both NDEs and GLOC experiences are those neurologic activities captured within the subconscious state. Various neurologic activities may occur within subconsciousness or lower neurologic states, but they would be unlikely to be captured in a state lower than subconsciousness. It follows that if indeed it is the subconscious state that is key to remembering these experiences, then the characteristics of the experiences are at least partially related to the duration within the subconscious state. Although the duration and depth of the ischemic insult that produces unconsciousness may have an effect on the experiences, we would not predict that experiences in the unconscious or lower states would become memorable. To be described to others, the experiences must be reportable. To be reportable, the nervous system must be in a state of consciousness. The neurologic states induced by $+\mathrm{Gz}$ stress are energy dependent, as illustrated in Figure 4. 


\section{Conclusions}

Solving the G-LOC problems in fighter aviation requires understanding the neurologic states of consciousness, subconsciousness, and unconsciousness, along with the mechanisms that cause the transitions between neurologic states. The same need for understanding is shared by those investigating NDEs. Loss and recovery of consciousness are events common to individuals who have G-LOC and to NDErs. From this perspective, G-LOC provides a model for understanding neurologic states that are shared in common with the NDE. I have previously classified G-LOC episodes based on the magnitude of the ischemic (energy deficit) insult to the nervous system to establish a framework for conducting G-LOC research safely (Whinnery, 1989a, 1991). The NDE can be included in this classification, based on physiologic insult similarity. This classification scheme has the potential for establishing a framework for the systematic study of the NDE and for relating near-death research to other psychophysiologic research efforts.

There are several types of alteration that can disturb the normal state of consciousness. There may well be similar neurologic shutdown and start-up mechanisms responsible for the transitions between consciousness and unconsciousness for different alteration etiologies. Although the kinetics of the loss and recovery of consciousness are different for hypoxia due to altitude exposure and ischemic hypoxia (G-LOC), many of the symptoms are similar. For instance, the enjoyable, lightheaded, euphoric feeling of altitude-induced hypoxia is not that different from the feelings that frequently result from G-LOC. These symptoms are shared by other mechanisms that also produce loss of consciousness. They have been pleasurable enough to cause addictive behavior for re-experiencing these symptoms (Lai and Ziegler, 1983) and to enhance sexual experience (Resnik, 1972). The loss of consciousness associated with the NDE, in comparison, has been described as resulting in an "elevated level of consciousness," transcendence, or a mystical state of consciousness. Indeed, it appears that many of the psychophysiologic symptoms reported following G-LOC episodes are remarkably similar to those of the NDE. As illustrated in Figures 3 and 4, G-LOC episodes and NDEs would be predicted to have commonality based on the shared neurologic state alterations that occur. The G-LOC/NDE common experience would be attributed to the psychophysiologic changes associated with loss and recovery of consciousness. The unique aspects 
of the NDE should become evident by comparison with the G-LOC symptoms.

The various alterations that lead to loss and recovery of consciousness can produce a wide spectrum of insults to the nervous system. The ischemic insults associated with G-LOC represent a narrow band within this overall spectrum. Although G-LOC in itself represents only a narrow band within the overall range of mechanisms that produce loss of consciousness, it results from a range of neurologic insults from minimal to severe. The symptoms observed reflect this range. G-LOC episodes represent a range within the minimal insult spectrum, while NDEs represent a range within the more severe neurologic insult spectrum. To gain command of the entire spectrum of symptoms that can result from various processes inducing loss and recovery of consciousness, it is necessary to compile the dispersed information that exists in the literature covering many disciplines. Loss and recovery of consciousness should be recognized as an integral part of the NDE and thoroughly investigated.

It is imperative that the most precise definitions be made of all terms pertaining to alterations of neurologic states, along with the most detailed descriptions of all symptoms and processes. Events described as "sudden death" can be misleading. If there is a spontaneous recovery and/or successful resuscitation, in which an individual regains a higher state of neurologic function than death, then the event was only a loss of consciousness episode. If the term "sudden death" is used to describe such events, then we would have to acknowledge that some individuals have died hundreds of times (Schwartz and Jezer, 1932). From a neurophysiologic perspective, such phenomena as sustained periods of cardiac asystole or ventricular fibrillation are not sudden death. They are just loss of consciousness episodes, albeit significant ones, since "no one has returned from the dead and given an account of his experience" (Noyes, 1972, p. 174). Although it may sound like a trivial statement, from a kinetic standpoint, if an organism is classified as dead, it must stay dead. If an organism regains higher neurologic states (above death), then it was not the state of death that the organism was in. It is for this reason that "near-death" rather then "sudden death" is much more appropriate terminology.

These altered neurologic state experiences, whether resulting from G-LOC or the NDE, are vivid reality to those who have them. Differences between G-LOC episodes and NDEs would be expected, if for no other reasons other than the circumstances that cause them 
and the magnitude of the insults to the nervous system. The G-LOC syndrome is the normal response of completely healthy individuals. If there are unique characteristics associated with the NDE, then their identification would be facilitated by focusing on the real differences in the individuals, the physical states, the environmental situation, and the symptomatology between G-LOC episodes and NDEs.

The situation associated with G-LOC is not life-threatening and is not perceived as a life-threatening situation by the individual. No resuscitation efforts other than removing the $+\mathrm{Gz}$ stress is needed for recovery from a G-LOC episode. The NDE frequently requires heroic efforts to restore consciousness. No panoramic memory or life review has been reported in association with G-LOC episodes. Panoramic memory is frequently reported in situations that are recognized by the individuals as being life-threatening (Noyes and Kletti, 1977). By itself, loss of consciousness does not appear to be the cause of panoramic memory. It is more common for the NDE to be associated with a life-threatening experience, and therefore more common for panoramic memory to be a part of the NDE. Identification of such differences is key to defining the unique symptom complex of the NDE.

Loss of consciousness episodes of all types appear to have an explainable physiologic basis. They are therefore open for scientific investigation. At least the loss of consciousness aspect of the NDE, therefore, has a potentially explainable basis. It would be odd if the symptoms associated with loss and recovery of consciousness were not part of the NDE. The fact that many of the NDE symptoms are similar to those resulting from loss and recovery of consciousness should suggest that individuals who report their NDEs have provided accurate symptom descriptions. This includes those symptoms beyond the scope of G-LOC experimentation, which are unique to the NDE.

\section{References}

Anonymous. (1969). Both ends of the stethoscope: Cardiac arrest. Lancet, 2, 262-264. Burton, R. R. (1988). G-induced loss of consciousness: Definition, history, current status. Aviation Space and Environmental Medicine, 59, 2-5.

Cammarota, J. P. (1992). Symptoms of acceleration (+Gz) induced incapacitation during pilot-in-the-loop simulated aerial combat. In Proceedings of the 1992 International Simulation Technology Conference and the 1992 Workshop on Neural Networks (pp. 58-63). San Diego, CA: Society for Computer Simulation. 
Dennett, D. C. (1993). Brainstorms: Philosophical essays on mind and psychology. Cambridge, MA: MIT Press.

Dlin, B. M, Stern, A., and Poliakoff, S. J. (1974). Survivors of cardiac arrest: The first few days. Psychosomatics, 15, 61-67.

Druss, R. G., and Kornfeld, D. S. (1967). The survivors of cardiac arrest: A psychiatric study. Journal of the American Medical Association, 201, 291-296.

Forster, E. M, and Whinnery, J. E. (1988). Recovery from Gz-induced loss of consciousness: Psychophysiologic considerations. Aviation Space and Environmental Medicine, 59, 517-522.

Hobson, J. A. (1988). The dreaming brain. New York, NY: Basic Books.

Lai, C.-W., and Ziegler, D. K. (1983). Repeated self-induced syncope and subsequent seizures: A case report. Archives of Neurology, 40, 820-823.

Noyes, R. (1972). The experience of dying. Psychiatry, 53, 174-184.

Noyes, R., and Kletti R. (1977). Panoramic memory: A response to the threat of death. Omega, 8, 181-194.

Resnik, H. L. P. (1972). Erotized repetitive hangings: A form of self-destructive behavior. American Journal of Psychotherapy, 26, 4-21.

Sabom, M. B., and Kreutziger, S. (1977). Near-death experiences. Journal of the Florida Medical Association, 64, 648-650.

Schwartz, S. P., and Jezer, A. (1932). Transient ventricular fibrillation: Clinical and electrocardiographic manifestations of syncopal seizures in patient with auriculoventricular dissociation. Archives of Internal Medicine, 50, 450-469.

Whinnery, J. E. (1989a). Defining risk in aerospace medical unconsciousness research. Aviation Space and Environmental Medicine, 60, 688-694.

Whinnery, J. E. (1989b). Methods for describing and quantifying +Gz-induced loss of consciousness. Aviation Space and Environmental Medicine, 60, 798-802.

Whinnery, J. E. (1989c). Observations on the neurophysiologic theory of acceleration $(+\mathrm{Gz})$ induced loss of consciousness. Aviation Space and Environmental Medicine, $60,589-593$.

Whinnery, J. E. (1990). The G-LOC syndrome (NADC Technical Report No. 91042-60). Warminster, PA: Naval Air Development Center.

Whinnery, J. E. (1991). Medical considerations for human exposure to acceleration-induced loss of consciousness. Aviation Space and Environmental Medicine, 62, 618623.

Whinnery, J. E. (In press). The brain in the black box (NAWC Report). Newport, RI: Naval War College.

Whinnery, J. E, and Burton, R. R. (1987). +Gz-induced loss of consciousness: A case for training exposure to unconsciousness. Aviation Space and Environmental Medicine, 58, 468-472.

Whinnery, J. E, and Jones, D. R. (1987). Recurrent +Gz-induced loss of consciousness. Aviation Space and Environmental Medicine, 58, 943-947.

Whinnery, J. E., and Whinnery, A. M. (1990). Acceleration-induced loss of consciousness: A review of 500 episodes. Archives of Neurology, 47, 764-776. 